\title{
Cost-effectiveness analysis of antiviral therapy in patients with advanced hepatitis B virus-related hepatocellular carcinoma treated with sorafenib
}

Authors: Pengfei Zhang ${ }^{1,2^{*}}$, Yu Yang ${ }^{1,2^{*}}$, Feng Wen ${ }^{1,2}$, John Wheeler ${ }^{3}$, Ping $\mathrm{Fu}^{2,4}$, Qiu $\mathrm{Li}^{1,2 \S}$

Authors' Affiliations: 1. Department of Medical Oncology, Cancer Center, State Key Laboratory of Biotherapy, West China Hospital, Sichuan University, China. 2.

West China Biostatistics and Cost-Benefit Analysis Center, Sichuan University, China. 3. School of Public Health, University of Michigan, Ann Arbor, Michigan 48109, USA. 4. Division of Nephrology, Department of Internal Medicine, West China Hospital, Sichuan University, China.

*These authors contributed equally to this work.

\section{Corresponding author:}

§Qiu Li, M.D., Ph.D., Department of Medical Oncology, Cancer Center, State Key Laboratory of Biotherapy, West China Hospital, Sichuan University; West China Biostatistics and Cost-Benefit Analysis Center, China. Tel: +86-28-85423262; Fax: +86-28-85423609; E-mail: keythera@126.com

This is the author manuscript accepted for publication and has undergone full peer review but has not been through the copyediting, typesetting, pagination and proofreading process, which may lead to differences between this version and the Version of Record. Please cite this article as doi: $10.1111 /$ jgh. 13425

This article is protected by copyright. All rights reserved. 


\section{Abstract}

Background and Aim: Antiviral therapy has been demonstrated to significantly improve the survival in patients with advanced hepatitis B virus (HBV)-related hepatocellular carcinoma (HCC). The aim of the study was to investigate the cost-effectiveness of antiviral therapy in patients with advanced HBV-related HCC treated with sorafenib.

Methods: To conduct the analysis, a Markov model comprising three health states (progression-free survival, progressive disease and death) was created. The efficacy data were derived from the medical records. Cost data were collected based on the Chinese national drug prices. Utility data came from the previously published studies. One-way sensitivity analyses as well as probabilistic sensitivity analyses were performed to explore model uncertainties.

Results: In the base-case analysis, addition of antiviral therapy to sorafenib generated an effectiveness of 0.68 QALYs at a cost of \$25026.04, while sorafenib monotherapy gained an effectiveness of 0.42 QALYs at a cost of \$20249.64. The incremental cost-effectiveness ratio (ICER) was \$18370.77/QALY for antiviral therapy group versus non-antiviral therapy group. On the other hand, the ICER between the two groups in patients with high or low HBV DNA load, with or without cirrhosis, normal or elevated alanine aminotransferase (ALT)/aspartate aminotransferase (AST) were \$16613.97/QALY, \$19774.16/QALY, \$14587.66/QALY, \$19873.84/QALY, \$17947.07/QALY and \$18785.58/QALY, respectively. 
Conclusions: Based on the cost-effectiveness threshold (\$20301.00/QALY in China), addition of antiviral therapy to sorafenib is considered to be a cost-effective option compared with sorafenib monotherapy in patients with advanced HBV-related HCC in

China from the patient's perspective.

Keywords: cost-effectiveness, antiviral therapy, hepatitis B virus, hepatocellular carcinoma, sorafenib

\section{Introduction}

Hepatocellular carcinoma (HCC) is the fifth most commonly diagnosed malignancy and the second leading cause of cancer death worldwide [1]. Hepatectomy, liver transplantation and radiofrequency ablation (RFA) are potentially curative treatments for patients with early stage HCC [2]. Unfortunately, most of the HCC patients are found incurable at the time of diagnosis due to tumor progression and underlying liver dysfunction [3]. Sorafenib has been approved for the treatment of HCC with vascular invasion and/or distant metastasis [4, 5]. Regardless of the multiple treatment alternatives mentioned above, the clinical outcome of advanced $\mathrm{HCC}$ is poor.

The incidence of HCC in China accounts for more than $50 \%$ of cases worldwide and chronic HBV infection has been established as the dominant cause [6]. In China, $85 \%$ of the HCC cases are HBV-related [7]. Some previous studies have revealed a high HBV virus load as the key prognostic factor for the progression and recurrence of $\mathrm{HCC}$ [8-11]. Recently, a series of studies have investigated the function of antiviral therapy in the management of HBV-related HCC, which demonstrated that antiviral therapy improved 
the survival of advanced HBV-related HCC patients when combined with sorafenib, especially in patients with high HBV-DNA level [12, 13].

However, these studies did not take long-term therapy costs into consideration, which might result in a substantial economic burden for advanced HBV-related HCC patients due to the antiviral therapy and the prolongation of survival. On the other hand, there have been a large number of studies focusing on the cost-effectiveness of antiviral treatments in chronic hepatitis B patients, which suggested antiviral therapy is a cost-effective option for patients with HBV infection [14-16]. Nevertheless, there has been no economic evaluation studying antiviral therapy for advanced HBV-related HCC patients treated with sorafenib. Is it a cost-effective strategy to give antiviral therapy combined with sorafenib for advanced HBV-related HCC patients? Therefore, the aim of the study was to conduct an economic model to evaluate the long-term clinical benefit and cost-effectiveness of antiviral therapy for advanced HBV-related HCC patients treated with sorafenib.

\section{Methods}

\section{Patients}

In order to conduct the analysis, the information of a cohort of patients was collected from the medical records of the Department of Medical Oncology, Cancer Center, West

China Hospital, Sichuan University (China): 1. confirmed advanced HCC (histologically or clinically confirmed); 2. Eastern Cooperative Oncology Group (ECOG) performance status (PS): 0-2; 3. Child-Pugh liver function class A/B; 4. 
treatment with sorafenib as first-line regimen from 2010 to 2013; 5. detectable hepatitis B surface antigen (HBsAg) positive; 6. without co-infection with other viruses (hepatitis A, C, D virus or human immunodeficiency virus (HIV). This retrospective study was approved by the Ethics Committee of West China Hospital, Sichuan University. Informed consent was obtained from the patients or her/his family members.

\section{Model structure}

A decision analytic Markov model was constructed to simulate clinical and economic outcomes of patients with HBV-related HCC treated with sorafenib. The model consisted of three mutually exclusive health states: progression free survival (PFS), progressive disease (PD) and death. The cycle length was 1 month and during each cycle, the patients either remained in their assigned health state or progressed to another health state as represented in Figure 1. Transition probabilities between health states were estimated according to the method described by previous studies [17].

\section{Cost data}

Potentially differential direct medical costs included the costs of sorafenib, antiviral drugs, transcatheter arterial chemoembolization (TACE) and tests (laboratory and radiological tests). The prices of sorafenib, antiviral drugs and TACE were obtained according to the Chinese national drug prices. The unit costs of laboratory and radiological tests were retrieved from West China Hospital, Sichuan University, China. Furthermore, the costs of grade 3/4 adverse events (AEs) were also includes in our analysis. In addition, we also considered the assistance programs in our study. In the 
assistance program for sorafenib, the patients paid for the costs of the first 3 months and then they got sorafenib for free until the occurrence of endpoints (PD, death, intolerance of AEs). All costs were converted to US dollars.

\section{Effectiveness data}

The effectiveness data of sorafenib group and sorafenib plus antiviral therapy group were extracted from the survival analysis of the patients collected from the medical records. Health outcomes were denoted in quality-adjusted life years (QALYs) gained and utility scores of Markov states were obtained from previously published studies [18].

\section{Cost-effectiveness analysis}

The cost-effectiveness analysis was conducted from the Chinese patient's perspective. The outcome of the cost-effectiveness analysis was measured as incremental cost-effectiveness ratio (ICER) of sorafenib plus antiviral therapy group compared with sorafenib monotherapy group, which was calculated as incremental costs divided by incremental effectiveness. Willingness to pay (WTP) in the model was set at $3 \times$ the per capita GDP of China (\$20,301.00/QALY) based on the WHO guidelines for cost-effectiveness analysis [19]. As the survivals in both groups were short, discount rates were not considered in the study.

\section{Subgroup analyses}

We also conducted subgroup analyses based on several essential clinical variables. The efficacy data of patients were also derived from the Kaplan-Meier survival curves of 
patients in these subgroups. The cost-effectiveness data of addition of antiviral therapy to sorafenib compared with sorafenib monotherapy in these subgroups were measured as the methods mentioned above.

\section{Sensitivity analyses}

To examine the impact of parameters uncertainty in our model, one-way sensitivity analyses were conducted. The results of the one-way sensitivity analyses were expressed as a tornado diagram. Furthermore, a probabilistic sensitivity analysis (PSA) based on a Monte-Carlo simulation of 1,000 patients was also conducted, the results of which were presented using cost-effectiveness acceptability curves and scatter plots diagrams.

Model creation and data analysis were conducted by TreeAge 2011 (TreeAge, Williamstown, Massachusetts, USA) and SPSS Statistics (IBM, Armonk, New York, USA).

\section{Results}

\section{Patients'clinical characteristics}

Of the total 92 patients fulfilled the inclusion criteria, 41 patients were from the antiviral group and 51 were from the non-antiviral group. The data of the patients in both groups, including gender, age, hepatitis e antigen (HBeAg), ECOG PS, Child-Pugh classification, Barcelona Clinic Liver Cancer (BCLC) stage, alpha fetoprotein (AFP), alanine aminotransferase (ALT)/aspartate aminotransferase (AST), total bilirubin (TBIL), liver cirrhosis and HBV DNA were analyzed and the results were shown in 
Table 1. The baseline characteristics were balanced between the two groups and there were no significant differences.

\section{Treatment}

The initial dose of sorafenib for advanced HCC was $400 \mathrm{mg}$ twice per day. In general, patients in the antiviral group received $94.2 \%$ of the full dosage of sorafenib and patients in the non-antiviral group received $90.2 \%$ of the full dosage of sorafenib. In the antiviral group, 20 patients were treated with lamivudine (LAM, $100 \mathrm{mg}$ per day), 8 patients were treated with adefovir dipivoxil (ADV, $10 \mathrm{mg}$ per day), 7 patients were treated with entecavir (ETV, $0.5 \mathrm{mg}$ per day) and 5 patients were treated with telbivudine (LdT, $600 \mathrm{mg}$ per day). Besides, there was one patients treated with adefovir dipivoxil (ADV, $10 \mathrm{mg}$ per day) plus entecavir (ETV, $0.5 \mathrm{mg}$ per day). The median course of antiviral treatment was 10.3 months (range: 1.7-28 months). During the treatment, there were only 2 patients developed viral resistance, one in entecavir group and the other in adefovir dipivoxil group. Adefovir dipivoxil (ADV, $10 \mathrm{mg}$ per day) and entecavir (ETV, $0.5 \mathrm{mg}$ per day) were added to the patients with entecavir resistance and adefovir dipivoxil resistance patients, respectively. Antiviral therapy did not increase adverse events to patients treated with sorafenib (Table 2).

\section{Health outcomes}

The survival data in both groups were presented as Kaplan-Meier survival curves in Figure 2. The median overall survival (mOS) was 12.2 months in antiviral group and 8.0 months in non-antiviral group. In terms of median progression free survival (mPFS), 
the data was 6.0 months in antiviral group and 4.5 months in non-antiviral group. The monthly transition probability between the Markov states and utility scores were presented in Table 2. Overall, the effectiveness was 0.68 QALYs in the antiviral group and 0.42 QALYs in non-antiviral group. The incremental effectiveness between the two groups was 0.26 QALYs.

\section{Cost}

$82.9 \%$ (34 of 41 ) of the patients in the antiviral group and $76.5 \%$ (39 of 51) of the patients in the non-antiviral group received assistance from the assistance programs. The monthly costs of sorafenib, antiviral therapy, TACE, tests and grade 3/4 AEs were shown in Table 2. During a life span time, patients in the antiviral group and non-antiviral group cost \$25026.04 and \$20249.64, respectively. The incremental costs were $\$ 4776.40$ between the two groups.

\section{Cost-effectiveness analysis}

According to the cost analysis and effectiveness analysis described above, the ICER between the antiviral group and non-antiviral group was \$18370.77/QALY (Table 2). Based on the cost-effectiveness threshold that was set in the study, addition of antiviral therapy to HBV-related patients treated with sorafenib was of great cost-effectiveness value.

\section{Subgroup analyses}

Furthermore, we conducted a series of subgroup analyses to investigate the cost-effectiveness of patients with different essential baseline characteristics (Table 3). 
Addition of antiviral therapy to sorafenib was suggested as a cost-effective regimen both in patients with low (ICER \$19774.16/QALY) or high HBV DNA load (ICER \$16613.97/QALY). Likewise, whether for patients with liver cirrhosis or without liver cirrhosis, addition of antiviral therapy to sorafenib was also likely to be a potentially cost-effective treatment. We also found similar results in patients with elevated (ICER \$18785.58/QALY) or normal ALT/AST (ICER \$17947.07/QALY). Thus, the subgroup analyses demonstrated that addition of antiviral therapy to sorafenib could improve the cost-effectiveness of sorafenib monotherapy regardless of above baseline characteristics.

\section{Sensitivity analyses}

To investigate the impact of the most influential variables on our results, one-way sensitivity analyses were conducted by varying the model parameters over their range of value $( \pm 30 \%)$. The most influential parameters in both the antiviral group and the non-antiviral group were shown in the tornado diagram (Figure 3). In the analyses, the key drivers on the results were duration of PFS state for antiviral group, duration of PFS state for non-antiviral group and duration of PD state for non-antiviral group. Parameters such as utility of PD state, price of sorafenib, costs of tests for antiviral group, utility of PFS state, costs of tests for non-antiviral group, costs of TACE for non-antiviral group, costs of TACE for antiviral group, costs of antiviral therapy before progression, duration of $\mathrm{PD}$ state for antiviral group, costs of antiviral therapy after progression and extra costs after progression were also important factors influencing our 
results. However, parameters including costs of AEs for antiviral group and costs of AEs for non-antiviral group had little impact on the robustness of the analysis.

The uncertainty of the cost-effectiveness analysis was also investigated by a probabilistic sensitivity analysis. The results of the analysis were presented in Figure 4 using cost-effectiveness acceptability curves. The acceptability curves showed that addition of antiviral therapy to sorafenib was a more cost-effective treatment in $82.2 \%$ of the simulations at the WTP threshold of \$20301.00/QALY. According to WTP threshold beyond $\$ 20301.00 / \mathrm{QALY}$, combination of antiviral therapy with sorafenib was also the better option compared with sorafenib monotherapy.

Moreover, scatter plots diagrams for our analysis also indicated that addition of antiviral therapy to sorafenib was a cost-effective option compared with sorafenib monotherapy when the WTP threshold was set at \$20301.00/QALY (Figure 4B).

We also tested the stability of our model from the societal perspective. Overall, patients in the antiviral group gained 0.68 QALYs at a cost of $\$ 57468.22$, while patients in the non-antiviral group cost $\$ 36233.30$ and got 0.42 QALYs. The incremental costs and effectiveness between the two groups were \$21234.92 and 0.26 QALYs, respectively. The ICER between the two groups was $\$ 81672.77 /$ QALY. Thus, from the societal perspective, addition of antiviral therapy to sorafenib was not demonstrated to be of cost-effectiveness value compared with sorafenib monotherapy.

\section{Discussion}

HCC is rampant in many countries around the world. The incidence of HCC is much 
higher in China than in any other country [20]. Although a series of treatments have improved the overall survival of HCC, the prognosis of advanced HCC is unsatisfactory. HBV has been well known as the key risk factor for HCC and a high serum HBV DNA load is associated with the progression of $\mathrm{HCC}$ in patients with chronic hepatitis B [8-11]. Recently, a series of articles suggested that the survival of advanced HBV-related HCC was significantly improved by using antiviral therapy $[12,13]$. However, all these studies are lacking of financial implications [21]. Sorafenib is the only molecular agent to treat advanced HCC while it has not been demonstrated to be a cost-effective drug in China [22], hence, it is of great importance to distinguish population who may benefit from the agent most, or, in other words, to make the agent more cost-effective.

In our study, addition of antiviral therapy to sorafenib improved the effectiveness of advanced HBV-related HCC by 0.26 QALYs when compared with sorafenib monotherapy (0.68 QALYs vs. 0.42 QALYs) with an incremental cost of $\$ 4776.40$ (\$25026.04 vs. \$20249.64). The ICER in our analysis was \$18370.77/QALY of antiviral group compared with non-antiviral group, which was lower than the WTP threshold-set in our study (\$20301.00/QALY). Thus, based on the results of our study, we have demonstrated that addition of antiviral therapy to sorafenib is likely to be a cost-effective option for patients with advanced HBV-related HCC in China.

HBV replication is associated with severe liver cirrhosis and dysfunction and the level of serum HBV DNA is an important risk factor for HCC [23]. A high level of HBV 
DNA in HCC patients independently predicted poor disease-free survival (DFS) and OS after surgical resection [8-11]. For HCC patients treated with sorafenib, the impacts of the HBV load and antiviral therapy on survival have demonstrated by some retrospective studies $[12,13]$. Antiviral therapy could improve OS of HBV-related HCC patients treated with sorafenib, especially in patients with high baseline HBV DNA load. In our study, the efficacy data were consistent with previous studies both in high baseline HBV DNA load group and low baseline HBV DNA load group. Addition of antiviral therapy to sorafenib was demonstrated to be an economic regimen for patients with advanced HBV-related HCC no matter the baseline viral load. The financial implication was improved more significantly in the high viral load group as the survival was prolonged much longer by antiviral therapy in the high HBV DNA load group than in the low HBV DNA load group. It could be interpreted as antiviral therapy could decrease HBV DNA replication, decrease the risk of liver failure and increases the chances of receiving more treatment modalities for HBV-related HCC.

A large part of patients with hepatitis B will develop cirrhosis and complications of end-stage liver disease [24]. Previous studies have shown the marked decrease in survival among patients with decompensated cirrhosis $[25,26]$. In our study, we have demonstrated that antiviral therapy may decrease the progression of cirrhosis, hepatic decompensation and prolong the survival of patients with advanced HBV-related HCC. This could be interpreted as antiviral therapy could suppresses HBV replication, normalizes liver function, reduces hepatitis necroinflammation and fibrosis in patients 
with chronic hepatitis B. Thus, addition of antiviral therapy to sorafenib could also improve the cost-effectiveness both in liver cirrhosis group and no liver cirrhosis group. Liver dysfunction has been also demonstrated as a prognostic factor for $\mathrm{HCC}$, the prognosis of unresectable HCC patients with impaired liver function is much worse [27, 28]. ALT/AST was primary indicators of liver function. Antiviral therapy could significantly improve the survival regardless of the ALS/AST status of these patients. Moreover, addition of antiviral therapy to sorafenib could improve the economic implication of sorafenib both in elevated ALT/AST group and normal ALT/AST group. This may be explained as antiviral therapy could decrease the viral load and improve liver function in patients with advanced HBV-related HCC [29]. On the other hand, given the improved liver function, these patients could become available to more treatment option.

It should be pointed out that cost-effectiveness analysis could be conducted from different perspectives, with the argument that which perspective should be used to enhance the accuracy and extend the application of the results of the analysis [30]. Thus, we also conducted our analysis from the societal perspective in the sensitivity analysis. However, the results from the societal perspective were not consistent with the results from the patient's perspective. The ICER was $\$ 81672.77 / \mathrm{Q} A L Y$ from the societal perspective, which was much higher than the cost-effectiveness threshold set in the study. Given the high price of sorafenib and the modest incremental effectiveness between the two groups, it was no wonder that addition of antiviral therapy to sorafenib 
was not a more cost-effective option compared with sorafenib monotherapy from the societal perspective.

It is essential that several limitations of this current study need to be addressed. First, as the data in our study were retrospectively collected from medical records, prospective randomized control trials are required to be further verified the role of antiviral therapy in improving the cost-effectiveness of sorafenib in patients with HBV-related HCC. Second, due to the lack of utility data for HCC patients in China, the data were obtained from literature previously published abroad, which may not reflect the Chinese situation exactly. In addition, the costs of the supportive care were not included in our analysis as the data were too complicated to calculate.

In conclusion, our analysis suggested that compared with sorafenib monotherapy, addition of antiviral therapy to sorafenib is a more cost-effective option for advanced HBV-related HCC patients in China. Moreover, we demonstrated that antiviral therapy can also improve the cost-effectiveness of sorafenib regardless of the HBV DNA load, with or without cirrhosis and liver function status. To the best of our knowledge, this is the first study to investigate the cost-effectiveness of antiviral therapy in patients with advanced $\mathrm{HBV}$-related HCC treated with sorafenib. Unlike those clinical trials, this analysis provided evidences for addition of antiviral therapy to the treatment of HBV-related HCC from an economic aspect and the results of the analysis can also make for the decision-making of the patients, the governments and the healthcare financial structures. 


\section{( \\ $=$ \\ ( \\ () \\ References}

1. Jemal A, Bray F, Center MM, Ferlay J, Ward E, Forman D. Global cancer statistics. CA Cancer J Clin. 2011; 61:69-90.

2. Bruix J, Sherman M, American Association for the Study of Liver Diseases. Management of hepatocellular carcinoma: an update. Hepatology. 2011; 53:1020-2.

3. Forner A, Llovet JM, Bruix J. Hepatocellular carcinoma. Lancet. 2012; 379:1245-55.

4. Llovet JM, Ricci S, Mazzaferro V, Hilgard P, Gane E, Blanc JF, et al. Sorafenib in advanced hepatocellular carcinoma. N Engl J Med. 2008; 359:378-90.

5. Cheng AL, Kang YK, Chen Z, Tsao CJ, Qin S, Kim JS, et al. Efficacy and safety of sorafenib in patients in the Asia-Pacific region with advanced hepatocellular carcinoma: a phase III randomised, double-blind, placebo-controlled trial. Lancet Oncol. 2009; 10:25-34.

6. Chen W, Zheng R, Zhang S, Zhao P, Zeng H, Zou X. Report of cancer incidence and mortality in China, 2010. Ann Transl Med. 2014; 2:61.

7. Ming L, Thorgeirsson SS, Gail MH, Lu P, Harris CC, Wang N, et al. Dominant role of hepatitis B virus and cofactor role of aflatoxin in hepatocarcinogenesis in Qidong, China. Hepatology. 2002; 36:1214-20.

8. Perz JF', Armstrong GL, Farrington LA, Hutin YJ, Bell BP. The contributions of hepatitis $\mathrm{B}$ virus and hepatitis $\mathrm{C}$ virus infections to cirrhosis and primary liver cancer worldwide. J Hepatol. 2006; 45:529-38.

9. Yang T, Lu JH, Zhai J, Lin C, Yang GS, Zhao RH, et al. High viral load is associated with poor overall and recurrence-free survival of hepatitis B virus-related 
hepatocellular carcinoma after curative resection: a prospective cohort study. Eur J Surg Oncol. 2012; 38:683-91.

10. Yu SJ, Kim YJ. Hepatitis B viral load affects prognosis of hepatocellular carcinoma. World J Gastroenterol. 2014; 20:12039-44.

11. Chen L, Zhang Q, Chang W, Du Y, Zhang H, Cao G. Viral and host inflammation-related factors that can predict the prognosis of hepatocellular carcinoma. Eur J Cancer. 2012; 48:1977-87.

12. Yang Y, Wen F, Li J, Zhang P, Yan W, Hao P, et al. A high baseline HBV load and = antiviral therapy affect the survival of patients with advanced HBV-related HCC treated with sorafenib. Liver Int. 2015; 35: 2147-54.

13. Xu L, Gao H, Huang J, Wang H, Zhou Z, Zhang Y, et al. Antiviral therapy in the improvement of survival of patients with hepatitis B virus-related hepatocellular carcinoma treated with sorafenib. J Gastroenterol Hepatol. 2015; 30: 1032-9.

14. Wu B, Li T, Chen H, Shen J.

Cost-effectiveness of nucleoside analog therapy for hepatitis B in China: a Markov analysis. Value Health. 2010; 13:592-600.

15. Zhang C, Ke W, Gao Y, Zhou S, Liu L, Ye X, et al. Cost-effectiveness analysis of antiviral therapies for hepatitis B antigen-positive chronic hepatitis B patients in China. Clin Drug Investig. 2015; 35: 197-209.

16. Buti M, Brosa M, Casado MA, Rueda M, Esteban R. Modeling the cost-effectiveness of different oral antiviral therapies in patients with chronic hepatitis B. J Hepatol. 2009; 51:640-6.

17. Purmonen T, Martikainen JA, Soini EJ, Kataja V, Vuorimen RL, Kellokumpu-Lehtinen PL. Economic evaluation of sunitinib malate in second-line treatment of metastatic renal cell carcinoma in Finland. Clin Ther. 2008; 30:382-92.

18. Cammà C, Cabibbo G, Petta S, Enea M, Iavarone M, Grieco A, et al. Cost-effectiveness of sorafenib treatment in field practice for patients with hepatocellular carcinoma. Hepatology. 2013; 57:1046-54.

19. Murray CJ, Evans DB, Acharya A, Baltussen RM. Development of WHO guidelines on generalized cost-effectiveness analysis. Health Econ. 2000; 9:235-51.

20. Chen JG, Zhang SW. Liver cancer epidemic in China: past, present and future. Semin Cancer Biol. 2011; 21:59-69.

21. Meropol NJ, Schulman KA. Cost of cancer care: issues and implications. J Clin Oncol. 2007; 25:180-6.

22. Zhang P, Yang Y, Wen F, He X, Tang R, Du Z, et al. Cost-effectiveness of sorafenib as a first-line treatment for advanced hepatocellular carcimoma. Eur J Gastroenterol Hepatol. 2015; 27:853-9.

23. Peng CY, Chien RN, Liaw YF. Hepatitis B virus-related decompensated liver cirrhosis: benefits of antiviral therapy. J Hepatol. 2012; 57:442-50. 
24. Fung SK, Lok AS. Management of patients with hepatitis B virus-induced cirrhosis. J Hepatol. 2005; 42 Suppl (1):S54-64.

25. Zipprich A, Garcia-Tsao G, Rogowski S, Fleig WE, Seufferlein T, Dollinger MM. Prognostic indicators of survival in patients with compensated and decompensated cirrhosis. Liver Int. 2012; 32:1407-14.

26. Shah NL, Banaei YP, Hojnowski KL, Cornella SL. Management options in decompensated cirrhosis. Hepat Med. 2015;7:43-50.

27. Garwood ER, Fidelman N, Hoch SE, Kerlan RK Jr, Yao FY. Morbidity and

= mortality following transarterial liver chemoembolization in patients with hepatocellular carcinomaand synthetic hepatic dysfunction.

Liver Transpl. 2013; 9:164-73.

28. Kubo S, Takemura S, Tanaka S, Shinkawa H, Nishioka T, Nozawa A, et al. Management of hepatitis B virus infection during treatment for hepatitis B virus-related hepatocellular carcinoma. World J Gastroenterol. 2015; 21:8249-55.

29. Koda M, Nagahara T, Matono T, Sugihara T, Mandai M, Ueki M, et al. Nucleotide analogs for patients with HBV-related hepatocellular carcinoma increase the survival rate through improved liver function. Intern Med. 2009; 48: 11-7.

30. Johannesson M, O'Conor RM. Cost-utility analysis from a societal perspective. Health Policy. 1997;39:241-53.

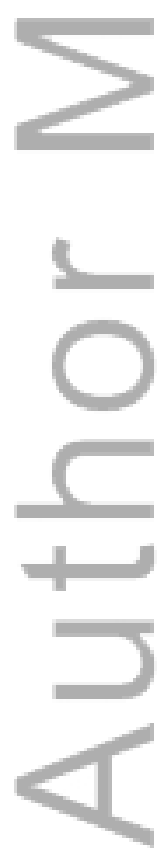

This article is protected by copyright. All rights reserved. 


\section{Figure Legends}

Figure 1 Markov model diagram for advanced hepatitis B virus (HBV) - related hepatocellular carcinoma (HCC).

Figure 2 Kaplan-Meier curves of PFS and OS.

A. Kaplan-Meier estimates of the progression-free survival of patients in the antiviral therapy group and non-antiviral therapy group.

B. Kaplan-Meier estimates of the overall survival of patients in the antiviral therapy group and non-antiviral therapy group.

Figure 3 Tornado diagram of the one-way sensitivity analysis for ICER.

ICER, incremental cost-effectiveness ratio; PFS, progression-free survival; PD, progressive disease; AEs, adverse events; QALY, quality adjusted life year; TACE, transcatheter arterial chemoembolization.

Figure 4 Cost-effectiveness acceptability curves and scatter plots diagrams of antiviral therapy group and non-antiviral therapy group.

A. Cost-effectiveness acceptability curves of antiviral therapy group and non-antiviral therapy group.

B. Scatter plots diagrams of antiviral therapy group and non-antiviral therapy group.

CE, cost-effectiveness; WTP, willingness-to-pay; QALM, quality-adjusted life month.

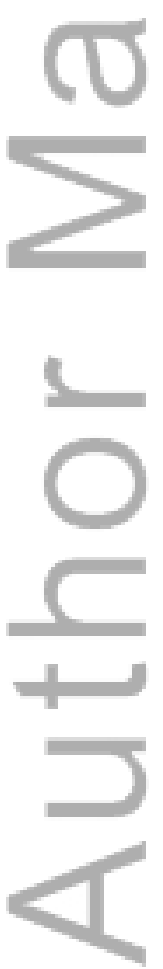

This article is protected by copyright. All rights reserved. 
Table 1 Baseline characteristics of HBV-related HCC patients treated with sorafenib.

\begin{tabular}{|c|c|c|c|c|}
\hline Variables & Total $(\mathrm{n}=92)$ & $\begin{array}{l}\text { Anti-viral } \\
\text { group }(n=41)\end{array}$ & $\begin{array}{l}\text { Non-antiviral } \\
\text { group }(n=51)\end{array}$ & $P$-value \\
\hline \multicolumn{5}{|l|}{ Age (years) } \\
\hline Median (range) & 49 (28-77) & $48(36-77)$ & $50(28-71)$ & 0.611 \\
\hline Male $[n(\%)]$ & $80(87.0)$ & $36(87.8)$ & $44(86.3)$ & 0.828 \\
\hline \multicolumn{5}{|l|}{ HB̈eAg } \\
\hline Positive [n (\%)] & 18 (19.6) & $9(22.0)$ & $9(17.6)$ & 0.605 \\
\hline Negative $[n(\%)]$ & $74(80.4)$ & $32(78.0)$ & $42(82.4)$ & \\
\hline \multicolumn{5}{|l|}{ ECOG PS } \\
\hline $0[\mathrm{n}(\%)]$ & $20(21.7)$ & $8(19.5)$ & $12(23.5)$ & 0.894 \\
\hline $1[\mathrm{n}(\%)]$ & $63(68.5)$ & $29(70.7)$ & $34(66.7)$ & \\
\hline $2[\mathrm{n}(\%)]$ & $9(9.8)$ & $4(9.8)$ & $5(9.8)$ & \\
\hline \multicolumn{5}{|l|}{ Child-Pugh class } \\
\hline$A[n(\%)]$ & $70(76.1)$ & $32(78.0)$ & $38(74.5)$ & 0.692 \\
\hline B [n (\%)] & $22(23.9)$ & $9(22.0)$ & $13(25.5)$ & \\
\hline \multicolumn{5}{|l|}{ BCLC stage } \\
\hline $\mathrm{B}[\mathrm{n}(\%)]$ & $27(29.3)$ & $13(31.7)$ & $14(27.5)$ & 0.656 \\
\hline $\mathrm{C}[\mathrm{n}(\%)]$ & $65(70.7)$ & $28(68.3)$ & $37(72.5)$ & \\
\hline \multicolumn{5}{|l|}{$\operatorname{AFP}(\mathbf{n g} / \mathrm{ml})$} \\
\hline$\leq 400[n(\%)]$ & 45 (48.9) & $19(46.3)$ & $26(51.0)$ & 0.658 \\
\hline$>400[n(\%)]$ & $47(51.1)$ & $22(53.7)$ & $25(49.0)$ & \\
\hline \multicolumn{5}{|l|}{ ALT/AST } \\
\hline Normal [n (\%)] & $28(30.4)$ & $12(29.3)$ & $16(31.4)$ & 0.827 \\
\hline Elevated [n (\%)] & $64(69.6)$ & $29(70.7)$ & 35 (68.6) & \\
\hline $\begin{array}{l}\text { TBIL } \\
\text { Normal [n (\%)] }\end{array}$ & $75(81.5)$ & $31(75.6)$ & $44(86.3)$ & 0190 \\
\hline Elevated [n (\%)] & $17(18.5)$ & $10(24.4)$ & $7(13.7)$ & \\
\hline \multicolumn{5}{|l|}{ Liver cirrhosis } \\
\hline Yes $[\mathrm{n}(\%)]$ & $43(46.7)$ & $23(56.1)$ & $20(39.2)$ & 0.107 \\
\hline No $[\mathrm{n}(\%)]$ & $49(53.3)$ & $18(43.9)$ & $31(60.8)$ & \\
\hline \multicolumn{5}{|l|}{ Pre-sorafenib HBV DNA } \\
\hline$\leq 10^{4}$ copies/ml [n (\%)] & $55(59.8)$ & $23(56.1)$ & $32(62.7)$ & 0.518 \\
\hline$>10^{4}$ copies/ml $[\mathrm{n}(\%)]$ & $37(40.2)$ & $18(43.9)$ & $19(37.3)$ & \\
\hline \multicolumn{5}{|l|}{ MVI } \\
\hline Yes $[\mathrm{n}(\%)]$ & $49(53.3)$ & $20(48.8)$ & $29(56.9)$ & 0.440 \\
\hline No [n (\%)] & $43(46.7)$ & $21(51.2)$ & $22(43.1)$ & \\
\hline \multicolumn{5}{|l|}{ Extrahepatic metastasis } \\
\hline Yes $[\mathrm{n}(\%)]$ & $23(25.0)$ & $11(26.8)$ & $12(23.5)$ & 0.716 \\
\hline
\end{tabular}

This article is protected by copyright. All rights reserved. 


\begin{tabular}{llccc}
\hline No [n (\%)] & $69(75.0)$ & $30(73.2)$ & $39(76.5)$ & \\
TACE & & & & \\
Yes $[n(\%)]$ & $32(34.8)$ & $15(36.6)$ & $17(33.3)$ & 0.746 \\
No $[\mathbf{n}(\%)]$ & $60(65.2)$ & $26(63.4)$ & $34(66.7)$ & \\
\hline
\end{tabular}

HBV, hepatitis B virus; HCC, hepatocellular carcinoma; HBeAg, hepatitis B e antigen; ECOG PS, Eastern Cooperative Oncology Group performance status; BCLC, Barcelona Clinic Liver Cancer; AFP, $\alpha$-fetoprotein; ALT, alanine aminotransferase; AST, aspartate aminotransferase; TBIL, total bilirubin; MVI; macroscopic vascular invasion; TACE, transcatheter arterial chemoembolization.

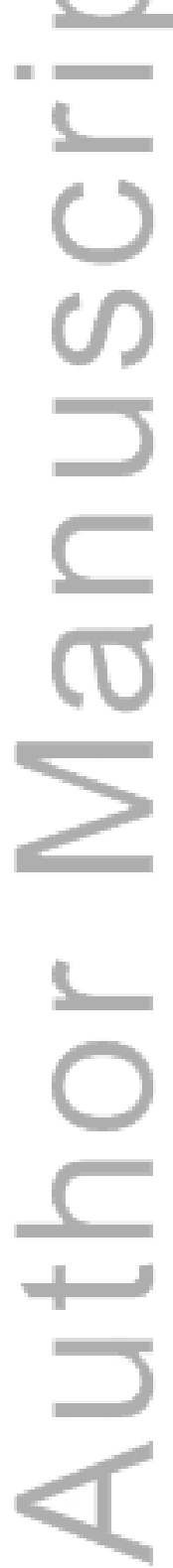

This article is protected by copyright. All rights reserved. 
Table 2 Base-case cost and effectiveness estimated.

\begin{tabular}{|c|c|c|c|c|}
\hline \multirow[b]{2}{*}{$\operatorname{AEs}[n(\%)]$} & \multicolumn{2}{|c|}{ Antiviral group } & \multicolumn{2}{|c|}{ Non-antiviral group } \\
\hline & Total & Grade 3/4 & Total & Grade $3 / 4$ \\
\hline HFS & $21(51.2)$ & $2(4.9)$ & $32(62.7)$ & $4(7.8)$ \\
\hline Diarrhea & $17(41.5)$ & $3(7.3)$ & $23(45.1)$ & $5(9.8)$ \\
\hline Fatigue & $10(24.4)$ & $0(0.0)$ & $9(17.6)$ & $0(0.0)$ \\
\hline Hypertension & $4(9.8)$ & $0(0.0)$ & $6(11.8)$ & $1(2.0)$ \\
\hline Anorexia & $10(24.4)$ & $2(4.9)$ & $12(23.5)$ & $2(3.9)$ \\
\hline Nausea & $9(22.0)$ & $1(2.4)$ & $10(19.6)$ & $2(3.9)$ \\
\hline \multicolumn{5}{|l|}{ Cost per month (\$) } \\
\hline Cost of sorafenib & \multicolumn{2}{|l|}{3643.93} & \multicolumn{2}{|l|}{4511.33} \\
\hline Antiviral cost before PD & \multicolumn{2}{|l|}{123.16} & \multicolumn{2}{|l|}{0.00} \\
\hline Cost of TACE & \multicolumn{2}{|l|}{133.42} & \multicolumn{2}{|l|}{195.02} \\
\hline Cost of tests & \multicolumn{2}{|l|}{182.10} & \multicolumn{2}{|l|}{187.06} \\
\hline AE-related costs & \multicolumn{2}{|l|}{4.51} & \multicolumn{2}{|l|}{6.68} \\
\hline Extra cost after PD & \multicolumn{2}{|l|}{102.50} & \multicolumn{2}{|l|}{102.50} \\
\hline Antiviral cost after PD & \multicolumn{2}{|l|}{58.00} & \multicolumn{2}{|l|}{0.00} \\
\hline Cost for the PFS state & \multicolumn{2}{|l|}{3953.70} & \multicolumn{2}{|l|}{4705.07} \\
\hline Costs for the PD state & \multicolumn{2}{|l|}{342.60} & \multicolumn{2}{|l|}{289.56} \\
\hline \multicolumn{5}{|l|}{ Utility } \\
\hline PFS & \multicolumn{2}{|l|}{0.76} & \multicolumn{2}{|l|}{0.76} \\
\hline PD & \multicolumn{2}{|l|}{0.68} & \multicolumn{2}{|l|}{0.68} \\
\hline Death & \multicolumn{2}{|l|}{0} & 0 & \\
\hline Transition probability & & & & \\
\hline $\mathbf{P}_{\text {PFS-PFS }}$ & 0.836 & & 0.774 & \\
\hline $\mathbf{P}_{\text {PFS }}$ & 0.109 & & 0.143 & \\
\hline $\mathbf{P}_{\text {PFS-death }}$ & 0.055 & & 0.083 & \\
\hline $\mathbf{P}_{\text {PD-PD }}$ & 0.894 & & 0.820 & \\
\hline $\mathbf{P}_{\text {PD-death }}$ & 0.106 & & 0.180 & \\
\hline Cost (\$) & & & & \\
\hline Cost for the PFS state & 22131.08 & & 18466.36 & \\
\hline Cost for the PD state & 2148.14 & & 1017.87 & \\
\hline Total cost & 25026.04 & & 20249.64 & \\
\hline Incremental cost & & 4776.40 & & \\
\hline Effectiveness (QALYs) & & & & \\
\hline Effectiveness for the PFS state & 0.32 & & 0.22 & \\
\hline Effectiveness for the PD state & 0.36 & & 0.20 & \\
\hline Total effectiveness & 0.68 & & 0.42 & \\
\hline Incremental effectiveness & & 0.26 & & \\
\hline
\end{tabular}

AEs, adverse events; HFS, hand-foot syndrome; PD, progressive disease; TACE, transcatheter arterial chemoembolization; PFS, 
progression free survival; QALYs, quality adjusted life years.

Table 3 Cost-effectiveness analysis of subgroups classified according to essential parameters.

\begin{tabular}{|c|c|c|c|c|c|c|}
\hline Variables & & Cost (\$) & $\begin{array}{l}\text { Incremental cost } \\
(\$)\end{array}$ & $\begin{array}{l}\text { Effectiveness } \\
\text { (QALYs) } \\
\end{array}$ & $\begin{array}{l}\text { Incremental effectiveness } \\
\text { (QALYs) }\end{array}$ & $\begin{array}{l}\text { ICER } \\
\text { (\$/QALYs) }\end{array}$ \\
\hline Baseline HBV DNA $\leq$ & Antiviral & 27781.86 & 4943.54 & 0.79 & 0.25 & 19774.16 \\
\hline $10^{4} \mathrm{cor}$ & Non-antiviral & 22838.32 & & 0.54 & & \\
\hline Baseline HBV DNA>10 $0^{4}$ & Antiviral & 26397.00 & 6147.17 & 0.71 & 0.37 & 16613.97 \\
\hline copies/mL & Non-antiviral & 20249.83 & & 0.34 & & \\
\hline With Liver cirrhosis & Antiviral & 24584.46 & 4668.05 & 0.70 & 0.32 & 14587.66 \\
\hline & Non-antiviral & 19916.41 & & 0.38 & & \\
\hline without liver cirrhosis & Antiviral & 25872.19 & 4968.46 & 0.78 & 0.25 & 19873.84 \\
\hline & Non-antiviral & 20903.73 & & 0.53 & & \\
\hline Elevated ALT/AST & Antiviral & 26200.60 & 4508.54 & 0.68 & 0.24 & 18785.58 \\
\hline & Non-antiviral & 21692.06 & & 0.44 & & \\
\hline Normal ALT/AST & Antiviral & 25538.12 & 4845.71 & 0.76 & 0.27 & 17947.07 \\
\hline 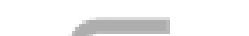 & Non-antiviral & 20692.41 & & 0.49 & & \\
\hline
\end{tabular}

QALYs, quality adjusted life years; ICER, incremental cost-effectiveness ratio; HBV, hepatitis B virus; ALT, alanine aminotransferase; AST, aspartate aminotransferase.

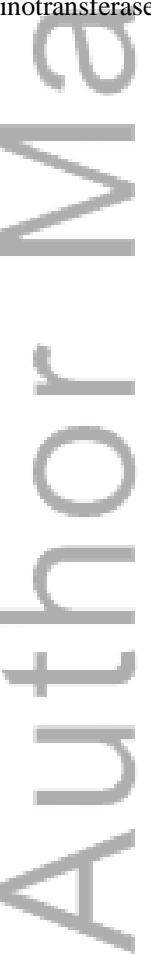

This article is protected by copyright. All rights reserved. 


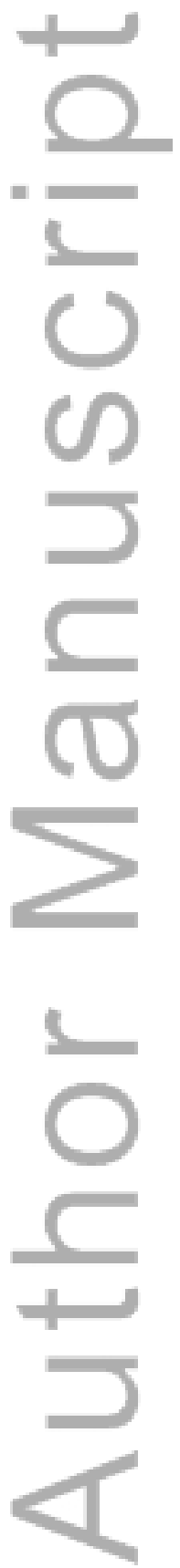

This article is protected by copyright. All rights reserved. 


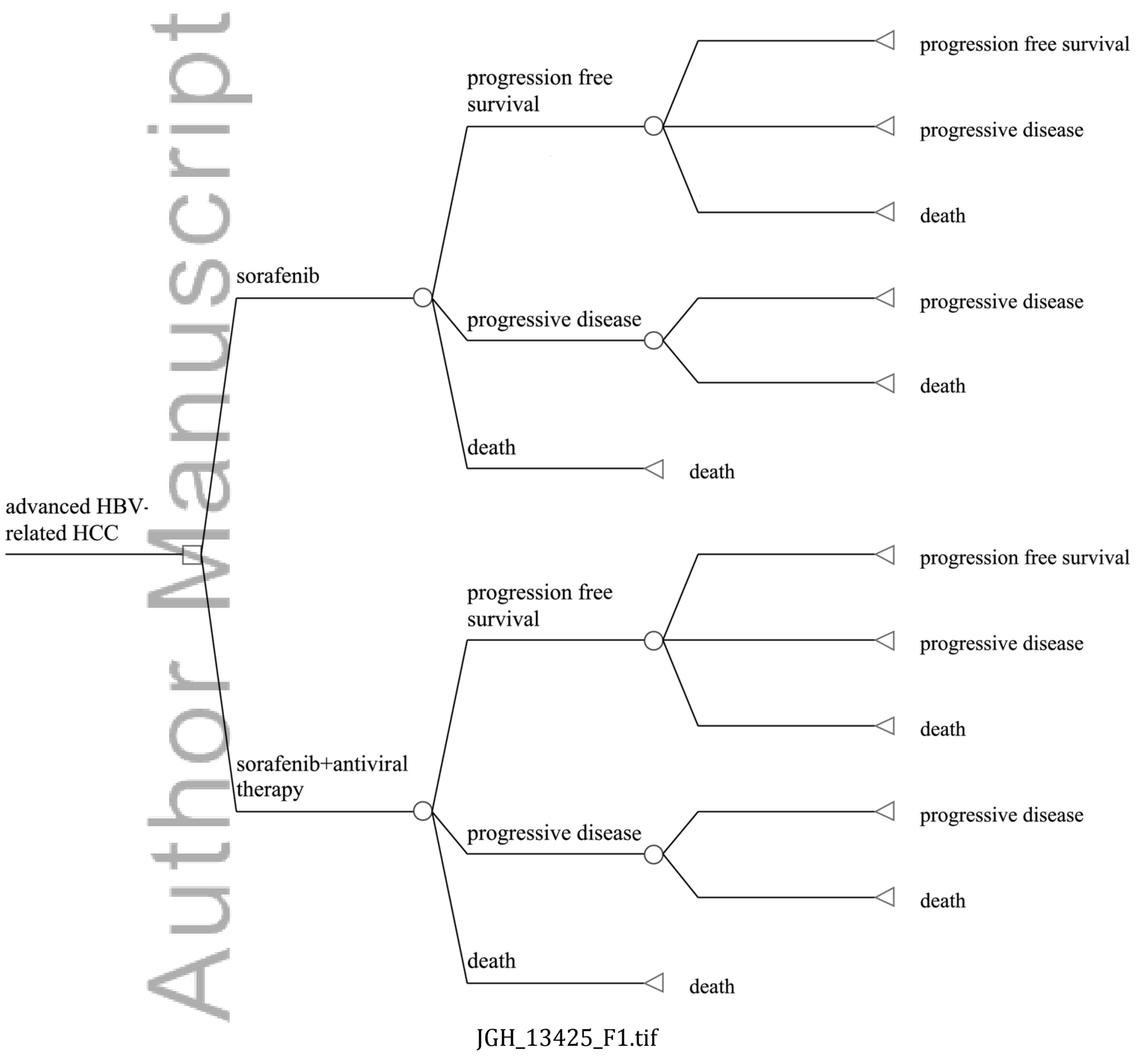

This article is protected by copyright. All rights reserved. 


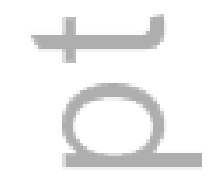

[

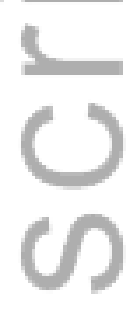

A

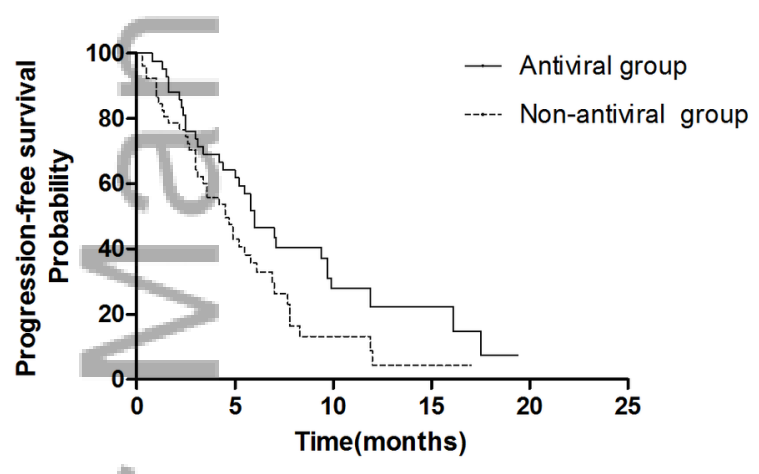

B

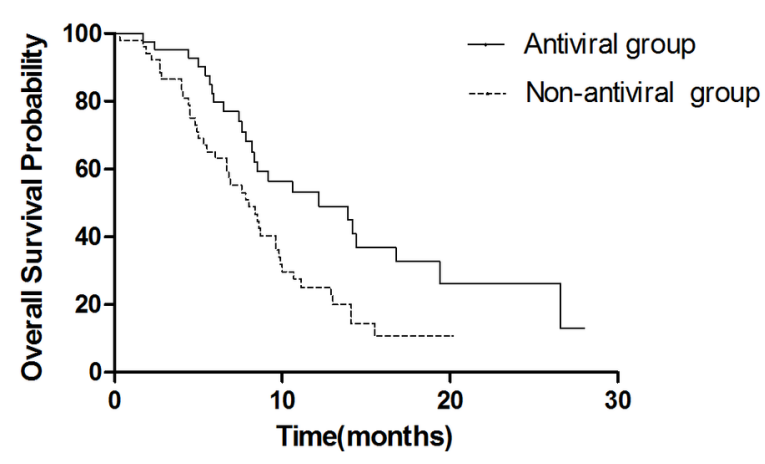

JGH_13425_F2.tif

This article is protected by copyright. All rights reserved. 


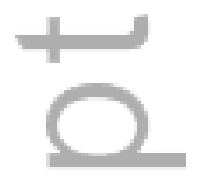

=

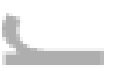

Duration of PFS state for antiviral group

Tornado Analysis (ICER)

Duration of PFS state for non-antiviral group Duration of PD state for non-antiviral group

Utility of PD state

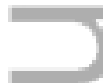

Price of sorafenib

Costs of tests for antiviral group

Utility of PFS state

Costs of tests for non-antiviral group

Costs of TACE for non-antiviral group

Costs of TACE for antiviral group

Costs of antiviral therapy before PD

Duration of PD state for antiviral group

Costs of antiviral therapy after PD

Extra costs after PD

Costs of AEs for non-antiviral group

Costs of AEs for antiviral group

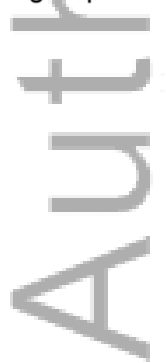

Base-Case: \$18370.77/QALY

JGH_13425_F3.tif

This article is protected by copyright. All rights reserved. 


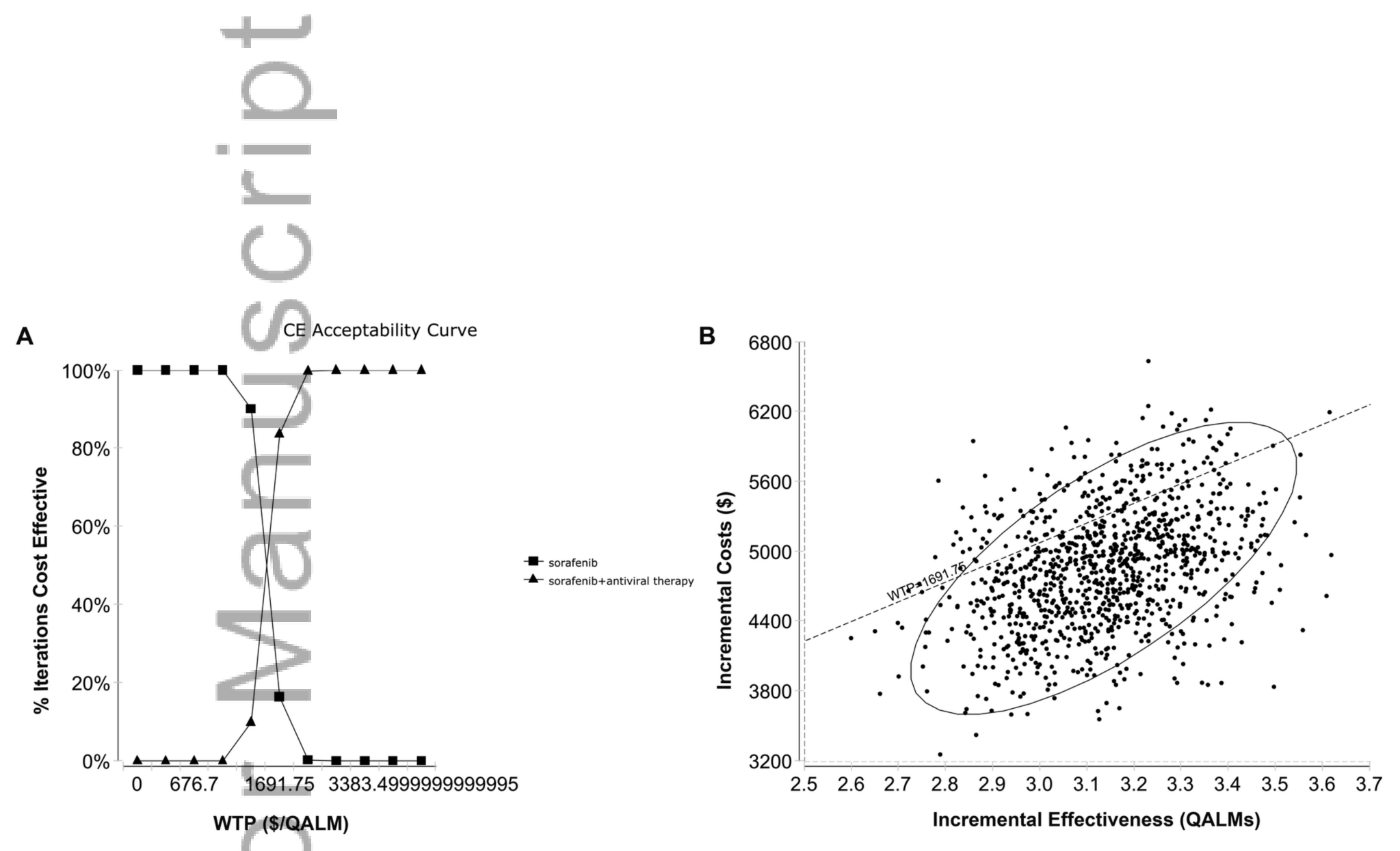

JGH_13425_F4.tif

This article is protected by copyright. All rights reserved. 